\title{
Generation of Pareto Frontier via a Modified Directed Search Domain Method
}

\author{
Tohid Erfani* and Sergei V.Utyuzhnikov ${ }^{\dagger}$ \\ The University of Manchester, School of Mechanical, Aerospace and Civil Engineering, \\ Manchester, M60 1QD, Great Britain \\ Brian Kolo $\ddagger$ \\ 11260, Zifian, Inc, Roger Bacon Dr, Reston, VA. 20190. United States
}

\begin{abstract}
Multiobjective optimization is one of the key challenges in engineering design process. Due to the non-uniqueness of the solution in this context, a set of evenly distributed solutions is particularly important for the designer. Directed Search Domain (DSD) method is proved to be efficient enough to tackle such a problem. In this paper, we introduce two main modifications of the DSD to make the algorithm simpler for application. They are related to the conduct of the search domain and reformulation of the appropriate single objective optimization problem. The proposed modifications increase the efficiency of the method in computational time with lower number of objective evaluations. A set of test cases demonstrates the capabilities of the new approach.
\end{abstract}

\section{Introduction}

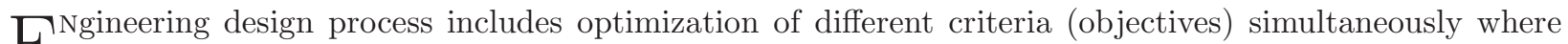
Ethe objectives are usually in conflict to each other. Therefore, the problem is to obtain a set of solutions which represents the best trade-off among the objectives. These solutions are called Pareto. Mathematically, a solution is a Pareto, if no improvement with respect to one objective can be made without worsening the quality of at least one of the others. In multiobjective optimization, Pareto solutions in objective space form a surface called the Pareto frontier. In literature, there are many methods which can be applied to obtain a single Pareto solution. However, only a few of them are capable of generating the whole Pareto frontier such as the Normal Boundary intersection (NBI) ${ }^{1}$ Normalized Normal Constraint $(\mathrm{NNC})^{2}$ and Directed Search Domain (DSD) $)^{3,4}$ methods. Each of these three algorithms is based on the anchor points which are the minimum points with respect to each objective. A set of evenly distributed points is generated on the hyperplane that contains all the anchor points and a single objective optimization is solved for each of them. This procedure leads to quasi even generation of the Pareto solutions at the Pareto frontier. The single objective optimization used for aforementioned methods are different. In particular, DSD introduces a search domain based on the local linear transformation of objectives and searches for the solution within each domain. To guarantee a well distributed Pareto set, it evenly spreads local search domains. However, this may bring some difficulties mainly due to introducing new coordinate systems.

In the general formulation cases and as recognized in, ${ }^{1}$ the above procedure may fail to capture the whole Pareto frontier in the cases with more than two objectives, if the projection of the Pareto surface onto the utopia hyperplane exceeds the utopia polygon. Although easy to be implemented, NBI method lacks the strategy to deal with this deficiency.

While NNC method extends the utopia hyperplane, DSD method uses a rotation strategy to deal with this problem and obtain the solution for the whole Pareto surface. The single objective formulation along the edges of the utopia hyperplane, in DSD algorithm, rotates towards the outer part of the plane in order to capture the solution in peripheral area.

\footnotetext{
*Post Graduate Researcher, AIAA Student Member, Email: tohid.erfani@postgrad.manchester.ac.uk

†Senior Research Fellow, School of MACE

$\ddagger$ Zifian Co.
} 
In this paper, we focus on the DSD method and introduce two modifications. Firstly, we change the formulation of the single objective optimization problem in a search domain and replace it by a simpler one. As a second task, we eliminate the rotation strategy and try to introduce a simple geometrical technique to define a new modified utopia hyperplane.

The paper is organized as follows. Section II provides the skeleton of the DSD method. The new modifications are made in Section III where we introduced the modfied DSD method. Then, in Section IV, we test the method on some challenging and well studied test problems. Finally, we summarize the methodology in Section VI.

\section{Directed Search Domain}

\section{A. Overview of DSD}

The algorithm contains the following steps. At first, the anchor points $\left(\mu_{i}\right)$ are generated for each objective function. Thereafter, the $\mu_{i}$ are used to form an interior of the utopia hyperplane $P$.

For each $M$ on $P$, the following single objective optimization should be solved

$$
\begin{array}{r}
\text { Min } \sum_{i=1}^{n} F_{i}(x), \\
\text { subject to } \quad F_{i} \leq M_{i}, \\
x \in \mathcal{D}^{*},
\end{array}
$$

where $\mathcal{D}^{*}$ is the feasible design space. The above constraint introduces a search domain where the solution should be searched for. Solving the above problem for each $M$ leads to a set of Pareto solutions; but not necessarily the evenly distributed one. To guarantee this, the search domain is shrunk by introducing a new coordinate system with the origin at a point $M$ and the axes forming angle $\theta$ with an arbitrary unit vector 1.

In the case of a non-convex boundary, it is possible not to obtain any feasible solution in the search domain. In this case, the search domain should be flipped to the opposite side of the utopia hyperplane to capture the points on the Pareto frontier. This is done by changing the $\leq$ to $\geq$ in equation 1 . In addition, to overcome the deficiency illustrated in Section I, the search domain is rotated if the point $M$ is located on the edge of the utopia hyperplane.

\section{B. DSD modifications}

The DSD method as illustrated in its original form has been used and tested on different challenging test cases and the results were promising. ${ }^{3}$ However, DSD can be improved considering the followings:

- Shrinking the search domain based on transformation from Cartesian to local coordinate system may be computational expensive.

- Flipping the search domain may not be efficient when the search space is highly oscillating.

- Rotation strategy may become hard to be implemented specially if the anchor points coincide.

In the interim of the paper, we address all of above issues and propose an improved version of DSD.

\section{DSD-II: algorithm sketch}

ShrinkIng AND FLIPPING In the proposed DSD-II, the shrinking constraint still exists. However, the shrinking procedure based on coordinate system transformation is replaced by the inner product of the two newly defined vectors. To realize this, we introduce $\nu$ as follows if the single objective optimization is run for point $M$ on $P$ :

$$
\nu=F-M
$$


where $F$ is the vector of objective function values of the current searching point $x$. In addition, the second vector $n$ is taken as the orthogonal vector to the utopia hyperplane $P$. Then, the shrinking in problem 1 can be described as

$$
\arccos \left(\frac{\nu \cdot n}{\|\nu\|\|n\|}\right) \leq \theta
$$

where the left hand side is the angle between $\nu$ and $n$ and (.) is the inner product between two vectors. By restricting the value for $\theta$, it is possible to shrink the search domain easier than before and generate the solution in the desirable area.

To overcome the computational cost inherent to the the flipping strategy, at each iteration, we calculate two angles using the above constraint as follows:

$$
\begin{gathered}
\gamma_{1}=\arccos \left(\frac{\nu \cdot n}{\|\nu\|\|n\|}\right) \leq \theta, \\
\gamma_{2}=\arccos \left(\frac{\nu \cdot-n}{\|\nu\|\|-n\|}\right) \leq \theta,
\end{gathered}
$$

where, $\gamma_{2}$ corresponds to the negative (flipped) direction of the normal vector $n$. Always, depending on the shape of Pareto surface, either $\gamma_{1}$ or $\gamma_{2}$ satisfies the shrinking constraint. Therefore,

$$
\min \left(\gamma_{1}, \gamma_{2}\right) \leq \theta
$$

is finally considered as the replacement for the existing shrinking constraint in problem 1.

Equation 3 obviously eliminates the first two mentioned difficulties. On the one hand it does not rely on the coordinate system and on the other hand the flipping is not required.

Rotating Rotation strategy in DSD is applied in two different situations. As stated earlier, one case is when the utopia hyperplane cannot cover the whole Pareto surface. The other occasion is the time when degeneration of the utopia hyperplane results in losing the normal direction $n$. Although there exist some guidelines to optimize the rotation strategy for the first case, ${ }^{4}$ in the second case, the rotation might be cumbersome and not even efficient. The problem which DSD faces in the second case, is similar for the NBI and NNC methods. To overcome this problem, we proceed as follows. We redefine the direction $n$ from the pseudo nadir point $N^{* 5}$ to the utopia point $U^{*}$ (the point which its components are the minimum of each objective). In the case of knowing the rough bounds of the objective space, one may take the direction from the worst point (possibly pseudo nadir) to the best point (possibly utopia).

$$
n=U^{*}-N^{*} .
$$

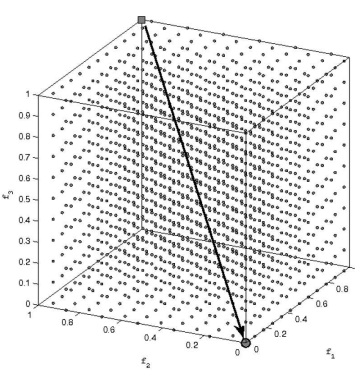

a

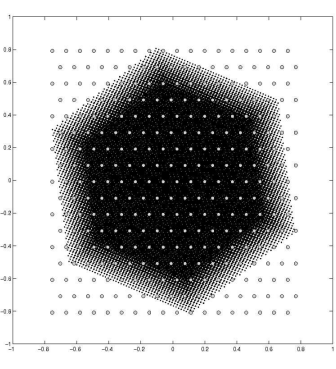

b

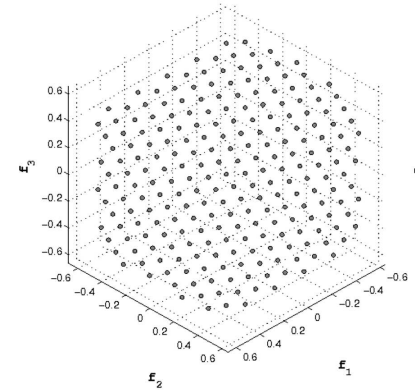

C

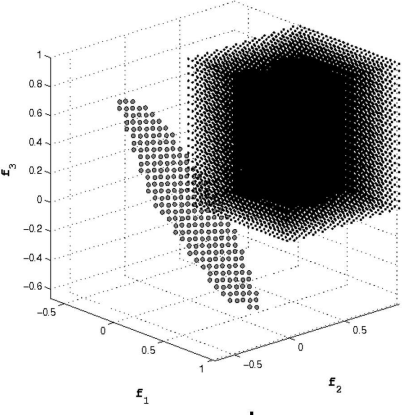

d

Figure 1. (a) the bounding box $C$, (b) $P P$, the black circles and $C P$, the grey circles, (c) $P$ with evenly generated points and $(d)$ the search box with the plane $P$ 
Then, a hypercube which limits the search space is defined by

$$
C=\left[f_{1}^{\min }, f_{1}^{\max }\right] \times\left[f_{2}^{\min }, f_{2}^{\max }\right] \times \ldots \times\left[f_{n s}^{\min }, f_{n s}^{\max }\right],
$$

where $n s$ is the number of objective functions. The maximum and minimum of each $f_{i}$ (objective functions) are taken from the utopia (best) and pseudo-nadir (worst) point. $C$ is a bounding hyper box with a uniform distribution of nodes within it. Next, the nodes $a \in C$ are projected to a hyperplane $P P$ with its normal vector defined by $n$ :

$$
P P=a^{t}-\frac{a^{t} n}{n^{t} n} n^{t}
$$

$P P$ guarantees that all the search space is covered. That is, there is no point in the hyper box $C$ with the possibility of being the solution on the space and be ignored. Obviously, projected points on $P P$ are not evenly distributed. To assure such a set, a covering hyperplane $C P$ with the same dimension as $P P$ is constructed by a mesh of uniformly distributed points on it. Then,

$$
P=C P \cap P P
$$

is obviously a hyperplane with the evenly distributed points $M$ on it. It should be noted that one may use rotation strategy ${ }^{3}$ instead of the above procedure. However, this mainly depends on the problem in the hand.

The schematic procedure for this step can be seen in Figure 1

With these new innovations, we present the DSD-II in Algorithm 1.

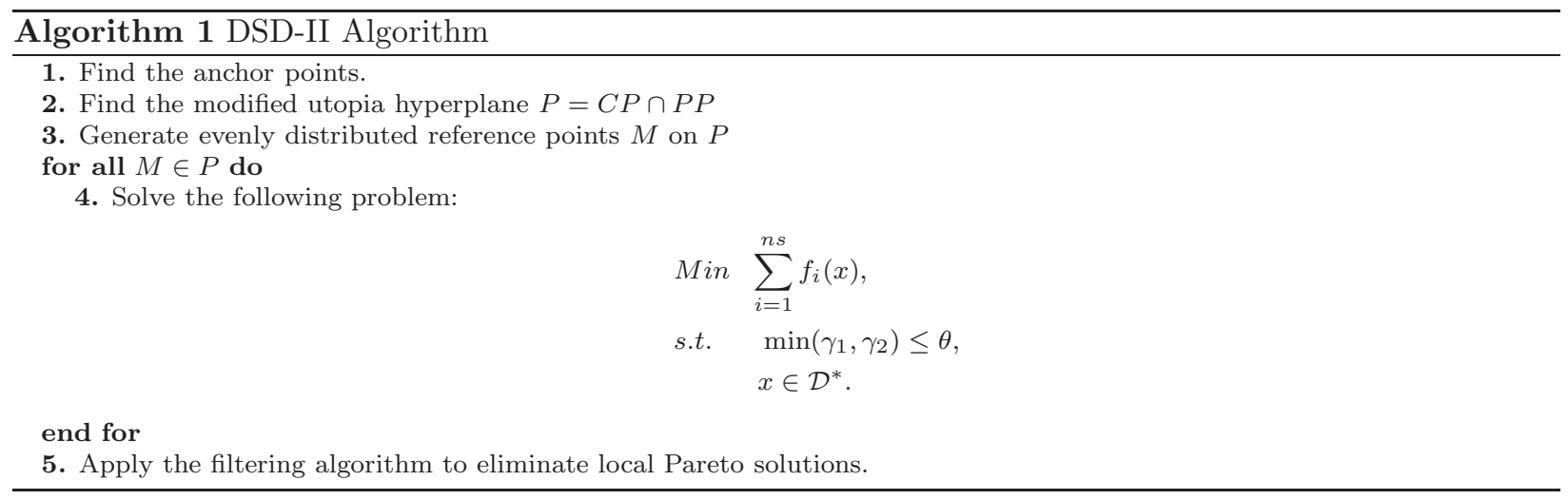

\section{Simulation results}

In this section, we demonstrate the performance of the DSD-II on the test cases. Test problems are chosen from a number of past studies in this area which presents a challenge to the multiobjective optimization.

\section{A. Performance Measures}

To evaluate the diversity of the solutions along the Pareto frontier, we use a coefficient of evenness, $E$, suggested by. ${ }^{4}$ In the case of a completely even set of solutions $E=1$. In addition, we report the number of objective function evaluations $O b$ for the sake of comparison with DSD method as well as the real time taken to solve the problem in seconds $(T i)$.

\section{B. Test problems}

SPIRAL This test case ${ }^{6}$ examines the efficiency of the method in obtaining the solution in highly oscillating Pareto surface. The results in Table 1 shows that not only DSD-II is timely more efficient than its older version, but also the computational cost has reduced by half. 


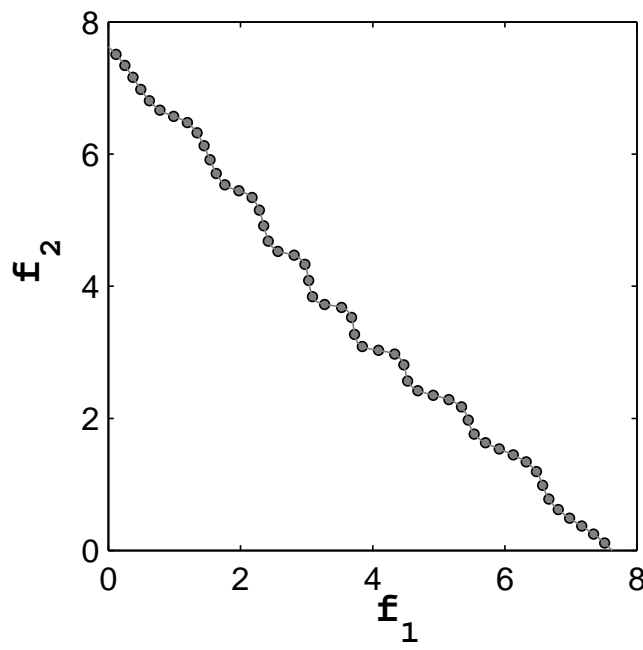

(a) Pareto solution for spiral using DSD-II

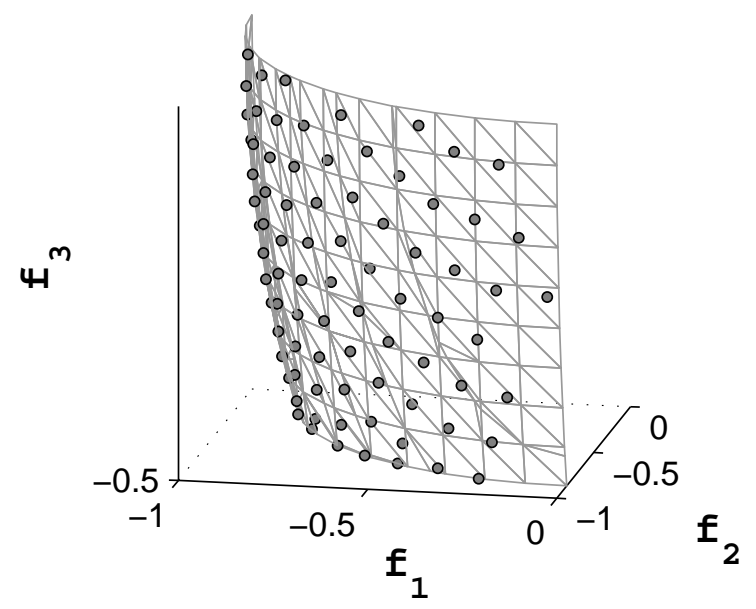

(b) Pareto solution for Tent using DSD-II

Figure 2. Pareto solutions using DSD-II

TENT In this test case proposed by, ${ }^{4}$ half of the Pareto surface can not be covered by the utopia hyperplane. Although the results do not indicate any significant change from that of DSD method, the difficulties in performing rotation strategy make DSD-II easier for implementation. It should be noted that NBI can not deal with such test case while the NNC is not efficient. ${ }^{4}$

DTLZ2 As reported in, ${ }^{7}$ NBI and NNC methods generate a number of redundant solutions for this three dimensional test case. As the Pareto surface is not convex, the number of objective evaluations are less in DSD-II compared to the DSD. Again, the rotation strategy is required by DSD while DSD-II does not need it.

DTLZ5 As the problem for all directional methods, this test case introduces the situation where the number of anchor points is less than the number of objective functions. Therefore, for the NNC and NBI, this cause a difficulty. DSD, as reported in, ${ }^{3}$ tackled this difficulty by its rotation and flipping strategy which comes with the cost of high number of objective evaluations. Although in DSD-II, also, some local solutions are generated and removed by the filtering, the approach does not rely on the flipping and rotation which make the implementation easier and more efficient.

\begin{tabular}{lcccccc}
\hline & \multicolumn{3}{c}{ DSD } & \multicolumn{3}{c}{ DSD-II } \\
\hline & $E$ & $O b$ & $T i(\mathrm{~s})$ & $E$ & $O b$ & $T i(\mathrm{~s})$ \\
\hline Spiral & 1.19 & 810 & 2.1 & 1.21 & $\mathbf{7 2 8}$ & $\mathbf{1 . 0}$ \\
Tent & 1.35 & 2210 & 5.5 & 1.40 & 2189 & 4.9 \\
DTLZ2 & 1.45 & 5410 & 5.0 & 1.38 & $\mathbf{4 6 2 0}$ & $\mathbf{3 . 5}$ \\
DTLZ5 & 1.42 & 2270 & 5.1 & 1.53 & $\mathbf{1 8 0 4}$ & $\mathbf{2 . 2}$ \\
\hline
\end{tabular}

Table 1. Comparison between DSD and DSD-II on $E$ (evenness), $O b$ (no. of objective evaluations) and $T i$ (real time spent in seconds) (bold shows significantly better performance) 


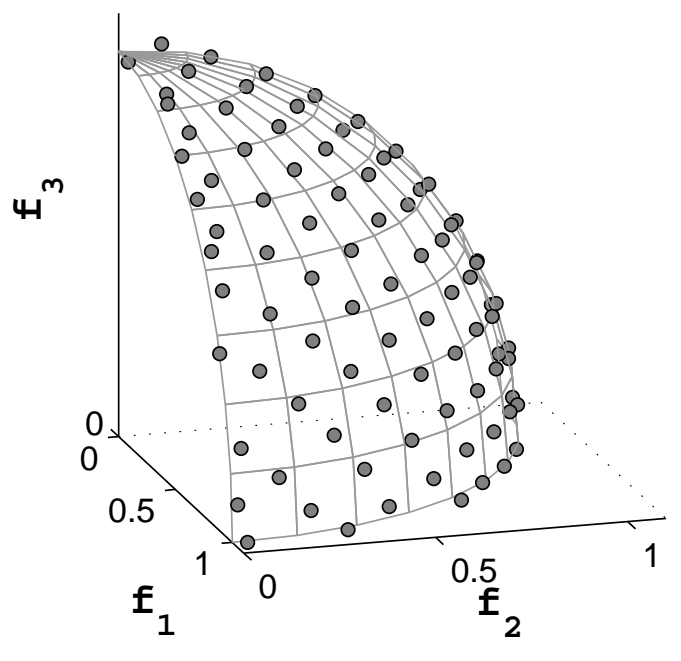

(a) Pareto solution for DTLZ2 using DSD-II

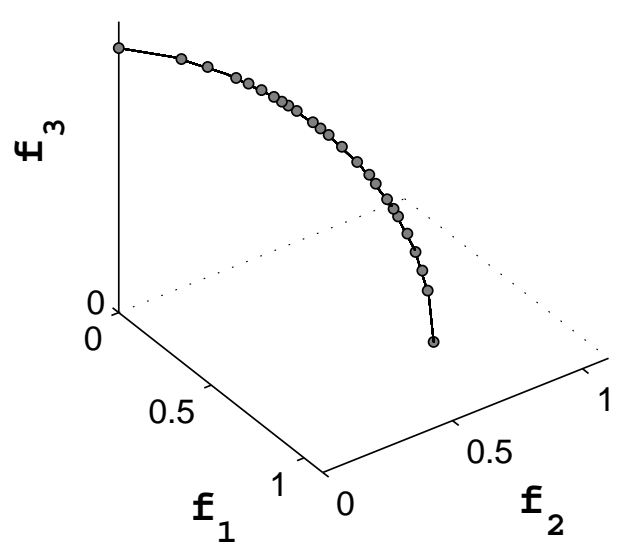

(b) Pareto solution for DTLZ5 using DSD-II

Figure 3. Pareto solutions for DTLZ test problems using DSD-II

\section{Further consideration}

\section{A. Ray emanation instead of parallel rays}

It has been shown that the modified utopia hyperplane reference points lead to a set of Pareto solutions. However, further investigations may result in easier approach. In this section, we propose another possible strategy to generate evenly distributed Pareto frontier which may be simpler for application. In this approach instead of evenly distributed set of reference points on (modified) utopia polygon, a set of ray emanating from utopia point is considered. Along each ray a single objective optimization is solved. To realize this, we proceed as follows.

First, the anchor points and the utopia point $U^{*}$ are obtained and the utopia polygon $P$ is constructed. Then, from the utopia point $U^{*}$ to each reference points $M$ on $P$, a ray $\left(\kappa_{1}\right)$ and searching vector $\left(\kappa_{2}\right)$ are formulated as

$$
\begin{gathered}
\kappa_{1}=M-U^{*}, \\
\kappa_{2}=F-U^{*} .
\end{gathered}
$$

Thereafter, the same optimization problem of Algorithm 1 is solved for each $M$ on $P$. This procedure 1) is easy to be implemented, 2) can cover the whole Pareto frontier and further, 3) does not require any scale mapping if the objectives are with different magnitudes. The method is illustrated in Fig. 4.

\section{Conclusion}

In this paper we have proposed DSD-II, a computationally efficient method in multiobjective optimization context. We consider three problems in which the existing methods in literature either fail to overcome or are not efficient in dealing with. By introducing a simple inner product as the shrinking strategy, we have replaced the shrinking procedure formerly implemented by the DSD method. This leads to elimination of the flipping in DSD algorithm which make the DSD-II more computationally efficient. In addition, the rotation strategy of DSD to capture the whole Pareto frontier has been eliminated. In turn, we have proposed a geometrical technique to generate the modified utopia hyperplane. The method is tested on different well

known test cases and results are compared with the DSD, NNC and NBI methods. The comparison shows that the DSD-II is more efficient yet simpler approach than the others. As a further consideration, we have 


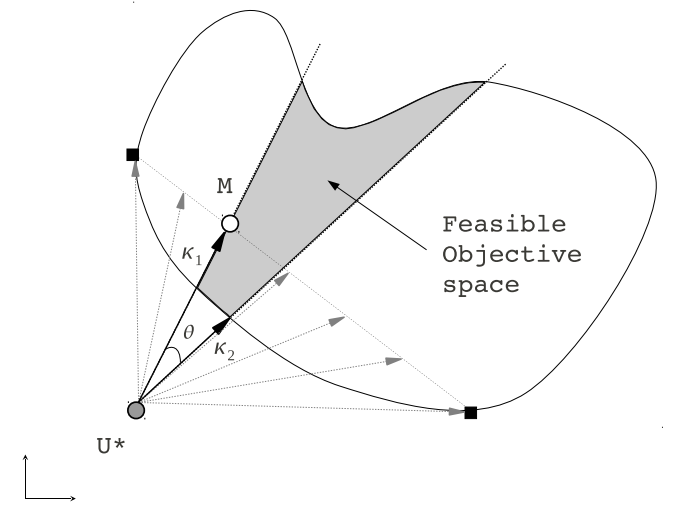

(a) A generic search space constructed by a ray for point $M$

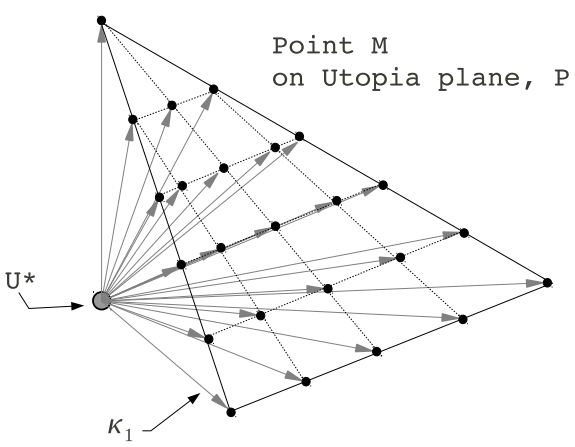

(b) Three dimensional ray emission strategy

Figure 4. Ray emanation for generating uniform spread of Pareto solution

introduced an alternative reference point generation technique by defining a set of rays emanating from the utopia point. These rays are used as the reference points to form the single objective sub-problems. It is concluded that both point generator approaches can be used interchangeably based on the problem under study. Still, DSD-II can be easily parallelized for faster computation.

\section{Acknowledgments}

The authors would like to thank the school of MACE at the University of Manchester for the financial support. The help and support of the administrative and technical staff of the University of Manchester are also greatly acknowledged.

\section{References}

\footnotetext{
${ }^{1}$ Das, I. and Dennis, J., "Normal-boundary intersection: A new method for generating the Pareto surface in nonlinear multicriteria optimization problems," SIAM Journal on Optimization, Vol. 8, 1998, pp. 631.

${ }^{2}$ Messac, A., Ismail-Yahaya, A., and Mattson, C., "The normalized normal constraint method for generating the Pareto frontier," Structural and Multidisciplinary Optimization, Vol. 25, No. 2, 2003, pp. 86-98.

${ }^{3}$ Erfani, T. and Utyuzhnikov, S., "Directed search domain: a method for even generation of the Pareto frontier in multiobjective optimization," Engineering Optimization, Vol. 43, No. 5, DOI:10.1080/0305215X.2010.497185, 2010, pp. 467-484.

${ }^{4}$ Utyuzhnikov, S., Fantini, P., and Guenov, M., "A method for generating a well-distributed Pareto set in nonlinear multiobjective optimization," Journal of Computational and Applied Mathematics, Vol. 223, No. 2, 2009, pp. 820-841.

${ }^{5}$ Miettinen, K., Nonlinear multiobjective optimization, Springer, 1999.

${ }^{6}$ Branke, J., Deb, K., Dierolf, H., and Osswald, M., "Finding knees in multi-objective optimization," Parallel Problem Solving from Nature-PPSN VIII, Springer, 2004, pp. 722-731.

${ }^{7}$ Deb, K., Thiele, L., Laumanns, M., and Zitzler, E., "Scalable test problems for evolutionary multiobjective optimization," Evolutionary Multiobjective Optimization, 2005, pp. 105-145.
} 